\title{
Anterior cage dislodgement in posterior lumbar interbody fusion: a review of 12 patients
}

\author{
Shuhei Murase, MD, ${ }^{1}$ Yasushi Oshima, MD, PhD, ${ }^{1}$ Yujiro Takeshita, MD, ${ }^{2}$ Kota Miyoshi, MD, PhD, ${ }^{2}$ \\ Kazuhito Soma, MD, ${ }^{1}$ Naohiro Kawamura, MD, PhD, ${ }^{3}$ Junichi Kunogi, MD, ${ }^{3}$ Takashi Yamazaki, MD, ${ }^{4}$ \\ Dai Ariyoshi, MD, ${ }^{5}$ Shigeo Sano, MD, PhD, ${ }^{5}$ Hirohiko Inanami, MD, ${ }^{6}$ Katsushi Takeshita, MD, PhD, \\ and Sakae Tanaka, MD, PhD ${ }^{1}$
}

\begin{abstract}
1Department of Orthopaedic Surgery, University of Tokyo; ${ }^{2}$ Department of Orthopaedic Surgery, Yokohama Rosai Hospital, Yokohama; ${ }^{3}$ Department of Spine and Orthopaedic Surgery, Japanese Red Cross Medical Center, Tokyo; ${ }^{4}$ Department of Orthopaedic Surgery, Musashino Red Cross Hospital, Musashino; ${ }^{5}$ Department of Orthopaedic Surgery, Sanraku Hospital, Tokyo; ' Department of Orthopaedic Surgery, Iwai Orthopaedic Medical Hospital, Tokyo; and 'Department of Orthopaedic Surgery, Jichi Medical University, Shimotsuke, Tochigi, Japan
\end{abstract}

\begin{abstract}
OBJECTIVE Interbody fusion cages are widely used to achieve initial fixation and secure spinal fusion; however, there are certain technique-related complications. Although anterior cage dislodgement can cause major vascular injury, the incidence is extremely rare. Here, the authors performed a review of anterior cage dislodgement following posterior lumbar interbody fusion (PLIF) surgery.
\end{abstract}

METHODS The authors retrospectively reviewed the cases of 4625 patients who had undergone PLIF at 6 institutions between December 2007 and March 2015. They investigated the incidence and causes of surgery-related anterior cage dislodgement, salvage mechanisms, and postoperative courses.

RESULTS Anterior cage dislodgement occurred in 12 cases $(0.26 \%)$, all of which were caused by technical errors. In 9 cases, excessive cage impaction resulted in dislodgement. In 2 cases, when the cage on the ipsilateral side was inserted, it interacted and pushed out the other cage on the opposite side. In 1 case, the cage was positioned in an extreme lateral and anterior part of the intervertebral disc space, and it postoperatively dislodged. In 3 cases, the cage was removed in the same operative field. In the remaining 9 cases, CT angiography was performed postoperatively to assess the relationship between the dislodged cage and large vessels. Dislodged cages were conservatively observed in 2 cases. In 7 cases, the cage was removed because it was touching or compressing large vessels, and an additional anterior approach was selected. In 2 patients, there was significant bleeding from an injured inferior vena cava. There were no further complications or sequelae associated with the dislodged cages during the follow-up period.

CONCLUSIONS Although rare, iatrogenic anterior cage dislodgement following a PLIF can occur. The authors found that technical errors made by experienced spine surgeons were the main causes of this complication. To prevent dislodgement, the surgeon should be cautious when inserting the cage, avoiding excessive cage impaction and ensuring cage control. Once dislodgement occurs, the surgeons must immediately address this difficult complication. First, the possibility of a large vessel injury should be considered. If the patient's vital signs are stable, the surgeon should continue with the surgery without cage removal and perform $\mathrm{CT}$ angiography postoperatively to assess the cage location. Blind maneuvers should be avoided when the surgical site cannot be clearly viewed. When the cage compresses or touches the aortic artery or vena cava, it is better to remove the cage to avoid late-onset injury to major vessels. When the cage does not compress or touch vessels, its removal is controversial. The risk factors associated with performing another surgery should be evaluated on a case-by-case basis.

https://thejns.org/doi/abs/10.3171/2016.12.SPINE16429

KEY WORDS complication; anterior cage dislodgement; vena cava injury; posterior lumbar spinal surgery; cage misplacement 
$\mathrm{T}$ ECHNOLOGICAL advances and good surgical results in posterior lumbar interbody fusion (PLIF) have made the procedure a popular choice for spine surgeons. However, complications associated with PLIF procedures that lead to poor surgical outcomes have been reported. Most perioperative cage misplacements are posterior or due to cage subsidence., $1,2,8,13$ Anterior cage dislodgement can cause serious problems because once the cage drops anteriorly, it becomes very difficult to maneuver and remove it from the same operative field. It may also be difficult to even identify the cage position. Injury to the aorta or inferior vena cava (IVC) could cause fatal complications. ${ }^{4,15,20,24}$ However, the incidence of anterior cage dislodgement is extremely rare, and few cases have been reported. ${ }^{3,6,7,21,22}$ Here, we report 12 cases of surgeryrelated anterior cage dislodgement; identify the cause, treatment, and postoperative course; and suggest how to deal with this complication based on our experience. To the best of our knowledge, this is the largest case series reported in the literature.

\section{Methods}

After receiving approval from the institutional review boards of 6 participating major spine referral centers, we retrospectively reviewed the cases of 4625 patients who underwent PLIF for lumbar degenerative diseases, such as spondylolisthesis, degenerative scoliosis, and lateral disc hernia, between December 2007 and March 2015. Our PLIF indications and procedures of each center were based on previous reports. ${ }^{5,9,16-18,23}$ We collected clinical data, operative notes, and imaging data for patients who had experienced perioperative anterior cage dislodgement. The incidence and causes of surgery-related anterior cage dislodgement, potential salvage mechanisms, and postoperative courses were investigated.

\section{Results}

Anterior cage dislodgement occurred in 12 patients ( $0.26 \%$; Table 1). In 9 cases, excessive cage impaction to attain optimal cage positioning in the anterior portion of the intervertebral disc resulted in dislodgement. In 2 cases, when the cage on the ipsilateral side was inserted, it interacted and pushed out the other cage on the opposite side. In 1 case, the cage was positioned in an extreme lateral and anterior part of the intervertebral disc space, which migrated and was found during radiographic examination on postoperative Day 4 before ambulation. Fluoroscopy control during cage placement was only used in 1 case.

The cage was immediately surgically removed from the same operative field in 3 cases. In the remaining 9 cases, CT angiography was performed after the operation to assess the location of the dislodged cage and whether large vessel or internal organ injury had occurred. In 7 of these cases, additional salvage surgery was performed via a retroperitoneal or transperitoneal approach. Eight patients underwent cage removal without any adverse events, and 2 patients sustained serious vascular injuries. The 2 patients were conservatively observed because CT angiography showed that the dislodged cages were in a relatively safe position with no vessel compression, and there were no further complications or sequelae associated with the cages during the follow-up period.

\section{Illustrative Cases \\ Case 1}

A 64-year-old woman with a history of degenerative scoliosis presented to the outpatient clinic with severe right low-back and leg pain that increased with walking. She underwent posterior fusion surgery from T-12 to L-5. A sublaminar taping system was placed at T-12, and a bilateral pedicle screw system was placed at L1-S1. Unilateral facetectomy and discectomy were performed at the right L2-3, L3-4, L4-5 and the left L5-S1 levels. The disc height at the right L2-3 level before surgery was $2 \mathrm{~mm}$ due to scoliosis. To modify the scoliosis, we had planned to place a 9-mm-high cage before the operation. After shaving, a maximal opening of the right L2-3 disc space was obtained using a spreader and a 9-mm trial spacer, and a

TABLE 1. Characteristics in patients who experienced anterior cage dislodgement in PLIF

\begin{tabular}{|c|c|c|c|c|c|c|c|}
\hline $\begin{array}{l}\text { Case } \\
\text { No. }\end{array}$ & $\begin{array}{l}\text { Age (yrs), } \\
\text { Sex }\end{array}$ & Diagnosis & $\begin{array}{l}\text { Migration } \\
\text { Level }\end{array}$ & Cause & $\begin{array}{l}\text { Approach for } \\
\text { Cage Removal }\end{array}$ & $\begin{array}{c}\text { Day of Cage } \\
\text { Removal }\end{array}$ & $\begin{array}{c}\text { Vascular } \\
\text { Injury }\end{array}$ \\
\hline 1 & $64, \mathrm{~F}$ & Degenerative scoliosis & L2-3 & Excessive impaction & Observation & None & None \\
\hline 3 & $81, F$ & Degenerative scoliosis & L4-5 & Ejection by opposite side's & Posterior & Intraop & None \\
\hline 4 & $56, \mathrm{~F}$ & Degenerative scoliosis & L3-4 & Ejection by opposite side's & Posterior & Intraop & None \\
\hline 5 & $80, M$ & Lat lumbar disc herniation & L4-5 & Excessive impaction & Retroperitoneal & 0 & None \\
\hline 7 & $72, \mathrm{~F}$ & Degenerative spondylolisthesis & L4-5 & Excessive impaction & Retroperitoneal & 12 & None \\
\hline 8 & $67, \mathrm{M}$ & Lumbar canal stenosis & L4-5 & Excessive impaction & Retroperitoneal & 33 & None \\
\hline 9 & $67, \mathrm{~F}$ & Degenerative scoliosis & L5-S1 & Excessive impaction & Transperitoneal & 13 & None \\
\hline 10 & $64, \mathrm{~F}$ & Degenerative scoliosis & L4-5 & Cage misplacement & Transperitoneal & 44 & None \\
\hline 11 & $85, \mathrm{M}$ & Lat lumbar disc herniation & $\llcorner 4-5$ & Excessive impaction & Posterior & Intraop & IVC injury \\
\hline
\end{tabular}


9-mm-high box-type cage was tapped into the disc space. After taking off the introducer and assessing the depth of the cage, more tapping to facilitate an optimal cage position was made using a final impactor, which was not locked with the cage and caused an anterior cage dislodgement. Intraoperative radiography showed that the cage was located in the anterior region. There was no severe bleeding from the retroperitoneal space, and the patient's vital signs were stable; therefore, cage removal was not attempted, and the operation was continued. Another cage was then meticulously inserted into the same L2-3 disc space, and the remaining cages were inserted at the right L3-4, L4-5, and left L5-S1 levels. The operation was completed without any further complications. Postoperative CT angiography performed immediately after the operation showed that the cage was near the right side of the IVC, but it did not seem to compress or indent any major blood vessel or internal organ. The patient was carefully monitored with CT angiography and radiographic examinations on a regular basis without the need for further surgical intervention. At the last follow-up at postoperative Month 36, there was only minimal movement change with cranial angulation, and there were no sequelae associated with the cage dislodgement (Fig. 1).

\section{Case 3}

An 81-year-old woman with a history of degenerative scoliosis presented to the outpatient clinic with complaints of neurogenic claudication and severe low-back pain. She underwent posterior fusion surgery at L4-5. A bilateral pedicle screw system was placed at L4-5, and a bilateral laminotomy and discectomy were performed at the L4-5 level. The disc height before surgery was $8 \mathrm{~mm}$. After shaving, a $10-\mathrm{mm}$ opening of the L4-5 disc space was obtained using a spreader, and 9-mm-tall box-type cages were inserted in both sides without any resistance. After bilateral cages were inserted into the L4-5 disc space, intraoperative radiography was performed that unexpectedly showed right cage dislodgement into the retroperitoneal region. The second left cage had pushed out the right cage that had already been inserted. The left cage was immediately removed, and arthroscopy was used to check the location of the dislodged cage and bleeding from the peritoneal space. Because an arthroscopic view provided no evidence of bleeding in the retroperitoneal space and accurate cage location, the dislodged cage was safely extracted under an arthroscopic view. Another cage was inserted, and the operation was completed without further incident. The patient's postoperative course was uneventful (Fig. 2).
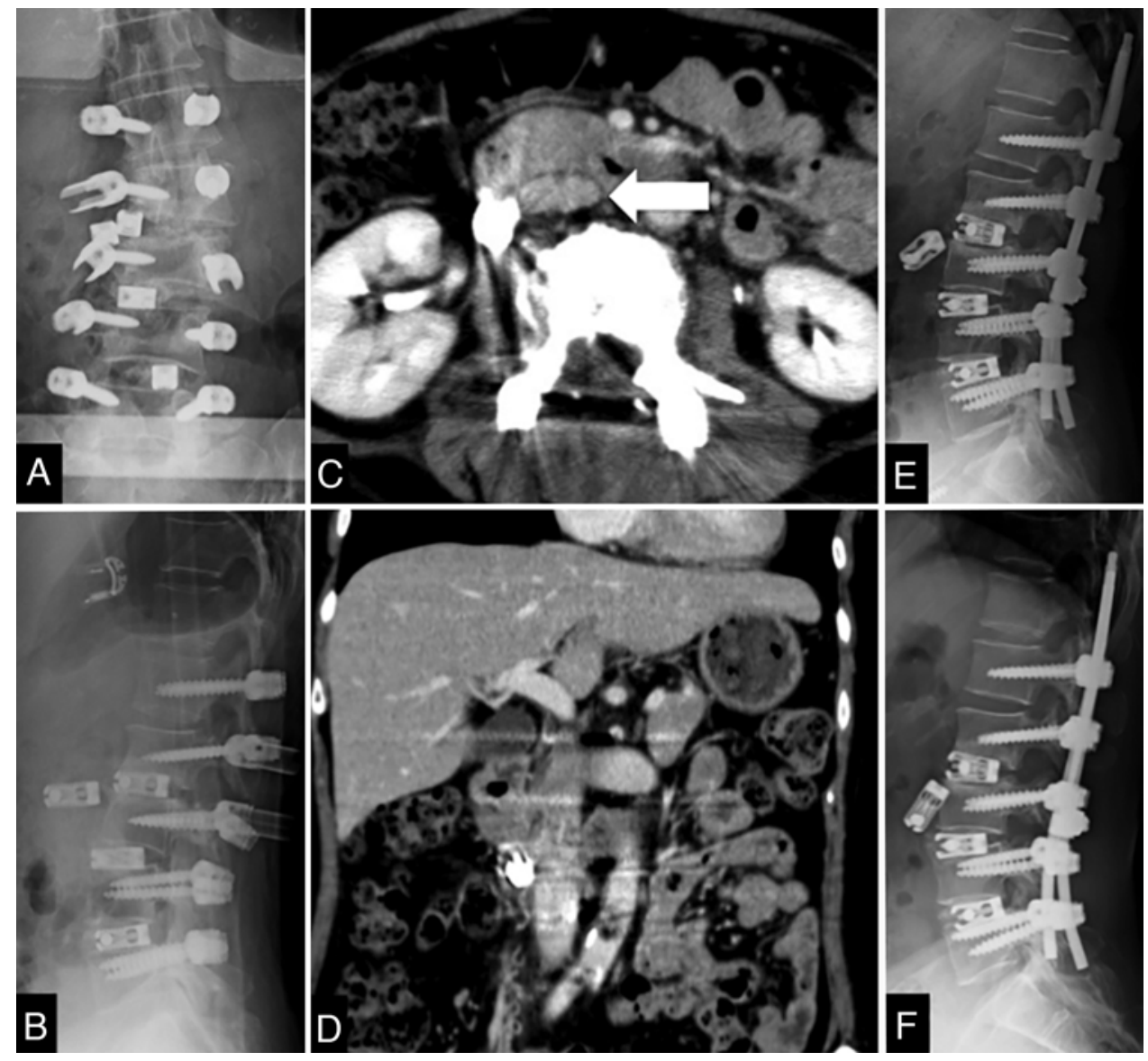

FIG. 1. Case 1. A and B: Intraoperative anteroposterior (A) and lateral (B) radiographs showing an L2-3-level anterior cage dislodgement. C and D: Postoperative CT angiograms showing the cage near the right of the IVC (arrow) but not compressing any major vessels. E and F: Radiographs obtained at discharge $(E)$ and last follow-up $(F)$, showing that the dislodged cage moved only slightly. 


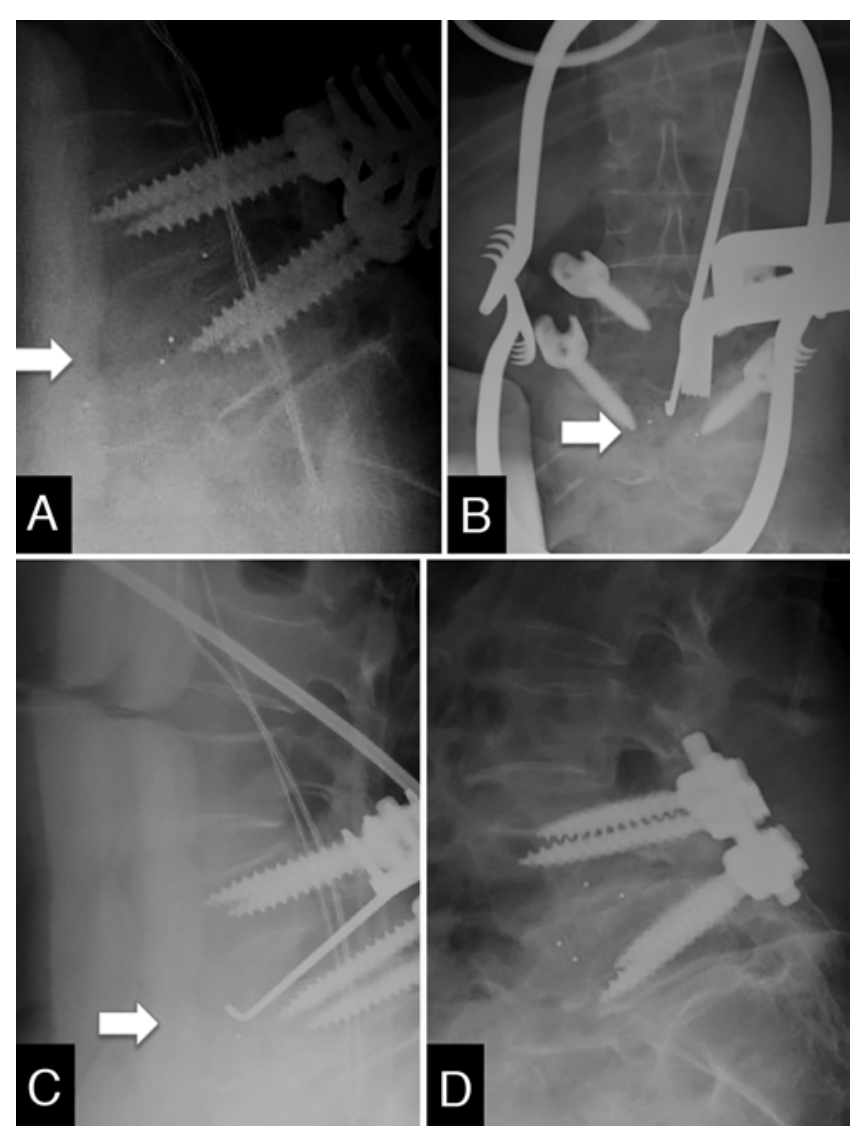

FIG. 2. Case 3. A: Intraoperative radiograph showing cage dislodgement (arrow). B and C: The hemorrhage was checked, and the cage was extracted using arthroscopy. The arrows indicate a probe that was used to maneuver the cage. D: After cage removal, the extracted cage was replaced, and the operation proceeded without event.

\section{Case 9}

A 67-year-old woman with a history of degenerative scoliosis presented with severe low-back and lower-extremity pain that increased with walking. She underwent posterior fusion surgery at L1-S1. A bilateral pedicle screw system was placed at L1-S1, and a unilateral facetectomy and discectomy was performed at the left L2-4 and right L4-S1 levels. After shaving and height opening of the disc space using a spreader and trial spacer, cage insertion at the left L2-4 and right L4-5 levels was performed without any incident. When the 9-mm-tall cage was inserted into the right L5-S1 disc space (the height before surgery was $6 \mathrm{~mm}$ ), excessive impaction by a final impactor to achieve an optimal cage position caused anterior cage dislodgement. During an attempt to capture the cage with its introducer, the cage slowly dislodged farther anteriorly. Fortunately, there did not appear to be any severe bleeding from the retroperitoneal space, and the patient's vital signs were stable. We decided not to remove the cage, and another cage was carefully inserted in its place. The operation was completed without further incident. Immediate postoperative CT angiography showed that the dislodged cage was very close to the right common iliac artery and compressing the IVC. The patient was transferred to another hospital, and the dislodged cage was removed via a transperitoneal approach on postoperative Day 13. The patient's postoperative course was uneventful (Fig. 3).

\section{Case 10}

A 64-year-old woman with a history of degenerative scoliosis presented to the outpatient clinic with severe low-back pain. Posterior spinal fusion surgery from L-1 to L-5 was performed. The preoperative L2-3, L3-4, and L4-5 disc heights on the concave side were approximately 5,7 , and $7 \mathrm{~mm}$, respectively, which we planned to extend. A bilateral pedicle screw system was placed at the L1-5 levels, and a bilateral laminectomy was performed at the L1-2, L2-3, L3-4, and L4-5 levels. After adequate disc preparation, 6-, 8-, and 8-mm disc height spreading and interbody spacer trial was achieved at the right L2-3, L3-4, and L4-5 disc spaces, respectively. The box-type cages with a similar spreading height were placed with a distraction force on the pedicle screws without incident. Postoperative radiography showed that the right L4-5 cage was placed too far laterally and anteriorly but was not dislodged. On postoperative Day 4, before ambulation, cage dislodgement into the peritoneal space was found on radiography. CT angiography showed that the cage remained close to the right common iliac vein and seemed to compress it. Although the patient underwent conservative follow-up without any complications, it was later decided that the cage should be removed via a transperitoneal approach. The patient's postoperative course was uneventful with no long-term sequelae (Fig. 4).

\section{Case 11}

An 85-year-old man presented to the outpatient clinic with a lateral lumbar disc herniation with degenerative spondylosis. He underwent posterior fusion surgery at L3-5. A bilateral pedicle screw system was placed at L35 , and a unilateral facetectomy and discectomy was performed at the right L4-5. The disc height before surgery was $4 \mathrm{~mm}$, and we planned to use a 7-mm-tall cage. After 7-mm height spreading and a spacer trial were performed, a 7-mm-high box-type cage was implanted and tapped through an introducer. Excessive impaction caused perforation of the anterior longitudinal ligament, which resulted in significant venous bleeding; the patient's blood pressure dropped to less than $60 \mathrm{~mm} \mathrm{Hg}$. The cage was promptly removed because it was still locked with the introducer. The source of bleeding could not be identified in the operative field; hemostatic materials were packed into the L4-5 disc space to control the bleeding. The patient's blood pressure was maintained using $4 \mathrm{U}$ of packed red blood cells and 6 $\mathrm{U}$ of fresh frozen plasma. The surgery proceeded, and the incision was closed in layers. Postoperative CT angiography showed an IVC injury, around which IVC hematomas, hemostatics, and migrated bone grafts were present. On postoperative Day 3, both legs, including the inguinal and gluteal regions, became swollen and the scrotum reached a size of $12 \mathrm{~cm}$. Angiography showed severe IVC compression and deep vein thrombosis. An IVC filter was inserted, and anticoagulation therapy with warfarin was initiated. The swelling gradually disappeared over the following 8 weeks. At the last follow-up at 29 months, the patient had no radicular pain or swelling (Fig. 5). 

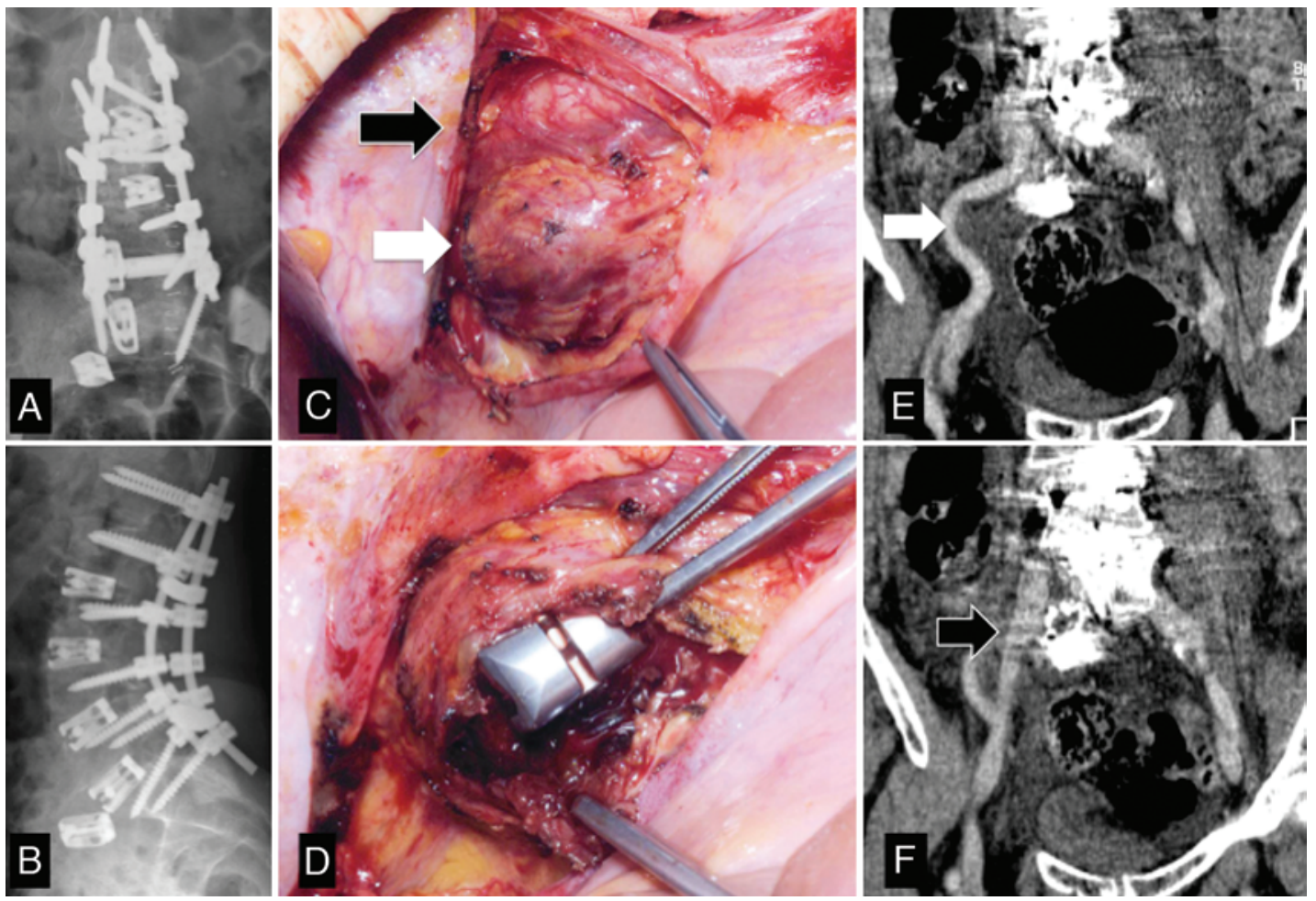

B

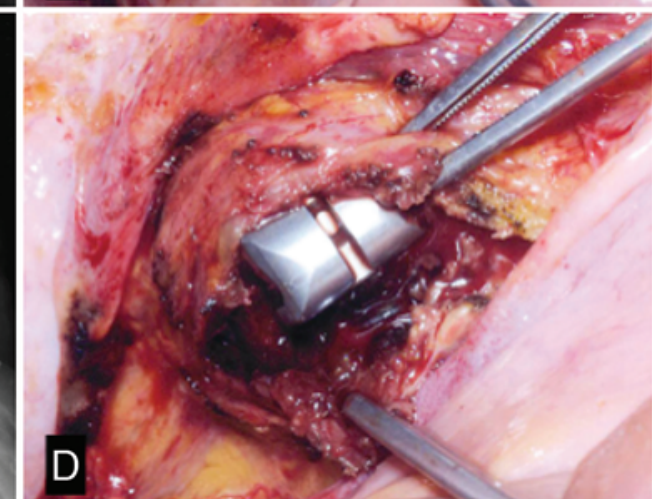

FIG. 3. Case 9. A and B: Intraoperative anteroposterior (A) and lateral (B) radiographs showing the right side of an L5-S1 anterior cage displacement. C and D: Intraoperative photographs showing the dislodged cage wrapped by aseptic fibrous tissue. The white arrow indicates the migrated cage; and the black arrow, the right ureter. E and F: Postoperative CT angiograms showing the dislodged cage in contact with the right common iliac artery (white arrow in E) and vein (black arrow in F). The dislodged cage was removed via a transperitoneal approach with no event. Figure is available in color online only.

\section{Discussion}

We report 12 cases of surgery-related anterior cage dislodgement that were adequately treated without serious sequelae. One of the most serious complications after cage dislodgement is a vessel injury, which occurred in 2 of our cases. If catastrophic bleeding occurs next to a dislodged cage, surgeons attempt to control the bleeding by packing the disc space with anticoagulant materials. In one case, the surgeon was able to control the bleeding using such a method and maintained circulatory dynamics without directly exploring the source of the bleeding. If bleeding cannot be controlled in this manner, surgeons must immediately turn the patient and detect the source of bleeding and control it via an anterior approach. ${ }^{20}$

If cage dislodgement has occurred with no significant bleeding and the patient's vital signs remain stable, then it is not an emergency, and the operation should be continued as planned. Postoperative CT angiography should be performed as soon as possible to check the cage position relative to large vessels, and then the surgeon must decide whether to remove the cage. When the cage does not compress large vessels or other intraabdominal organs, observation is an option. Ceylan et al. ${ }^{6}$ reported 3 cases of conservative treatment for cage dislodgement and claimed that early cage removal was not necessary unless an intraabdominal organ or vascular injury developed. However, there are some cases in which a misplaced screw or migrated bone grafts have caused compression and irritation, resulting in a postoperative deep vein thrombosis or aortic pseudoaneurysm, and the risk of leaving a dislodged cage in place remains unclear. ${ }^{10-12,14,19,25}$ Therefore, we recommend that a dislodged cage should be removed even if large vessels and intraabdominal organs are not compressed. We should evaluate the risk factors associated with performing another operation on a case-by-case basis and include data such as patient's medical history, age, and physical status.

Removing the cage from the same posterior surgical field is an approach that was performed in 3 of our cases. Arthroscopy was used to check the cage position and safety in 2 of these cases. However, in the remaining case, the cage was removed promptly, and the IVC injury was accompanied by significant bleeding. When the surgical view is not clear, blind maneuvers should be avoided, and anterior salvage surgery should be considered.

The other IVC injury occurred while performing an anterior salvage. In this case, the cage had dislodged into the IVC, and a laceration occurred during cage removal. It resulted in clamping of the IVC and both common iliac veins. Thus, we emphasize the importance of including a vascular surgeon on the surgical team. In this way, he/she can assist with the anterior portion of the procedure and contribute to planning the procedure if a major vascular injury is suspected..$^{3}$

In our cases, all cage dislodgements were due to technical errors, the most common of which was excessive cage impaction (9 cases). Anterior longitudinal ligaments at- 

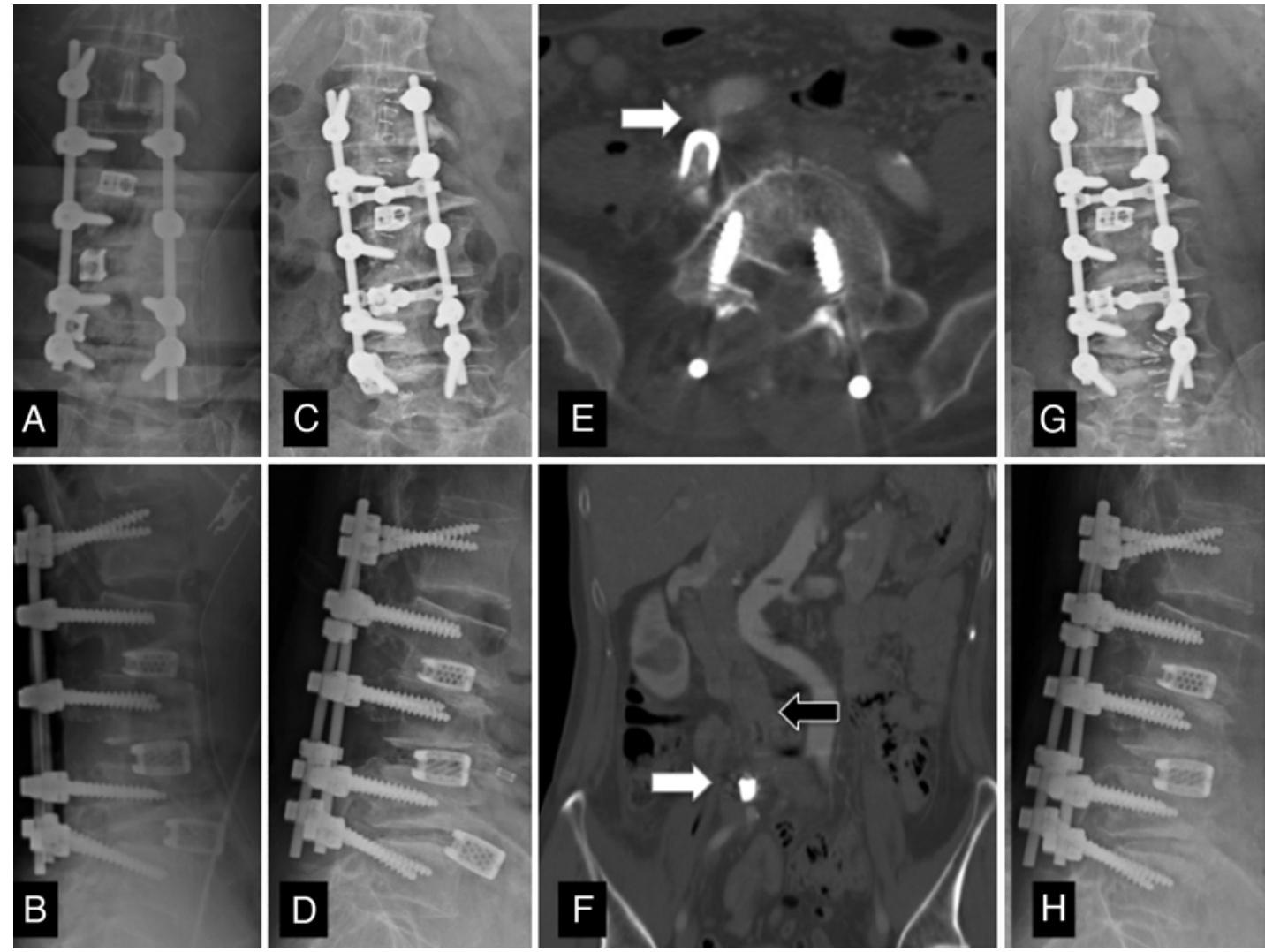

FIG. 4. Case 10. A and B: Anteroposterior (A) and lateral (B) radiographs obtained immediately postoperatively, showing that the cage is positioned too far laterally and anteriorly with no cage dislodgement. C and D: Anteroposterior (C) and lateral (D) radiographs obtained on postoperative Day 4, showing cage dislodgement. $\mathrm{E}$ and F: CT angiograms showing the dislodged cage near the right common iliac vein and compressing it. The white arrow indicates the right common iliac artery; and the black arrow, the IVC. G and H: Anteroposterior $(\mathrm{G})$ and lateral $(\mathrm{H})$ radiographs obtained after the second operation, showing the cage removal.

tach firmly to the anterior surfaces of the vertebrae and are not strong on the lateral side. Therefore, the cage should not be pushed too much when attaching it to the lateral part of the intervertebral disc space. Cage dislodgement is more likely to happen when a box cage is used instead of a boomerang cage. One of the underlying factors of this complication is degenerative scoliosis, where rotation of the vertebrae can lead to an anatomical disorientation of the surgical field. Surgeons should pay close attention in such cases. We should also be cognizant of the patient's osteoporotic condition, which could lead to a fracture of the endplate of the ventral disc attached by the ligaments and subsequent cage dislodgement. An oversized cage to gain maximal disc height needs powerful impaction that tends to cause excessive impaction, and a severe stretch of disc space results in the destruction of the anterior longitudinal ligament and the ventral disc endplate. In most cases we used cages that were almost the same size as the disc height. Measuring the disc height by $\mathrm{CT}$ or radiography before the operation is needed. If insertion of an oversized cage is required or planned, then careful cage insertion is necessary. Another error was the misdirected impaction of the second cage, which occurred in 2 of our cases. When the intervertebral disc space is small, the first box cage is suspected to be in the center of the intervertebral disc space, and it is difficult to insert 2 cages, the surgeon should not insert the second cage. In these 2 cases, the surgeons could have avoided or paid attention to the risk of anterior cage dislodgement preoperatively by measuring the intervertebral disc space.

Intraoperative fluoroscopy was only used in 1 case. Without fluoroscopy control, we have to unlock the introducer from the cage to assess the cage position by a depth gauge, and then perform impaction with a final impactor that is not locked to the cage. We believe that this leads to uncontrolled impaction. Therefore, fluoroscopy control could be useful to prevent excessive final impaction to achieve an optimal cage position. However, cage dislodgement occurred in 1 of our cases, and Ceylan et al. reported 3 cage dislodgements under fluoroscopy control. ${ }^{6}$ We must keep in mind that fluoroscopy control does not prevent anterior cage dislodgement perfectly.

To prevent anterior cage dislodgement caused by technical errors, we suggest noting some key points prior and during the procedure. First, it is important to make a detailed plan before surgery and check the small intervertebral disc space or for degenerative scoliosis. Second, although intraoperative fluoroscopy is helpful, it cannot be used in every case. In cases in which there is anatomical disorientation, not only the lateral view but also the anteroposterior view are useful in preventing lateral misplacement. Third, the use of an oversized cage should be 


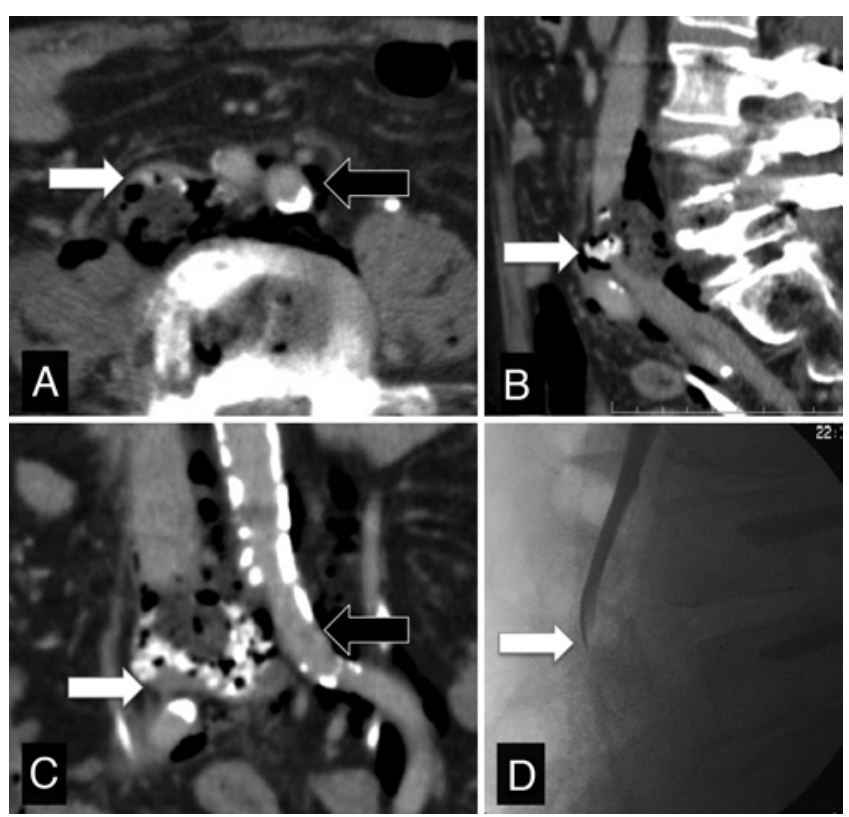

FIG. 5. Case 11. L4-5 cage dislodgement occurring during insertion. A-C: CT scans showing direct removal of the cage using an introducer. Bleeding was controlled by packing the disc space with Gelfoam and Surgicel. The white arrows indicate the common iliac vein being crushed by an enormous mass of hemostats; and the black arrows, the common iliac artery. D: Postoperative angiogram showing disruption of the IVC (arrow).

avoided because it requires strong impaction, and controlling the force is difficult. Sufficient shaving, distraction of the intervertebral disc space, and performing a cage trial would be useful to avoid excessive cage insertion. We have to bear in mind that severe stretch of the disc space could lead to the destruction of the anterior longitudinal ligament and ventral disc endplate. Finally, most cage dislodgements occurred during final impaction after detaching the holder. Therefore, we should endeavor to set appropriate cage positions before detaching the holder and avoid using a final impactor.

\section{Limitations}

This was a multicenter retrospective study. There were some differences regarding PLIF indications and procedures between the centers, although they were fundamentally based on previous reports.

\section{Conclusions}

Surgeons should keep in mind that anterior cage dislodgement could occur in every case. Our study revealed that technical errors by experienced spine surgeons were the main causes of this complication. Once it occurs, the possibility of large vessel injury should be considered. We hope that our study will help others prevent or cope with this serious complication.

\section{References}

1. Abbushi A, Cabraja M, Thomale UW, Woiciechowsky C, Kroppenstedt SN: The influence of cage positioning and cage type on cage migration and fusion rates in patients with monosegmental posterior lumbar interbody fusion and posterior fixation. Eur Spine J 18:1621-1628, 2009

2. Aoki Y, Yamagata M, Nakajima F, Ikeda Y, Takahashi K: Posterior migration of fusion cages in degenerative lumbar disease treated with transforaminal lumbar interbody fusion: a report of three patients. Spine (Phila Pa 1976) 34:E54E58, 2009

3. Ariyoshi D, Sano S, Kawamura N: Inferior vena cava injury caused by an anteriorly migrated cage resulting in ligation: case report. J Neurosurg Spine 24:409-412, 2016

4. Bingol H, Cingoz F, Yilmaz AT, Yasar M, Tatar H: Vascular complications related to lumbar disc surgery. J Neurosurg 100 (3 Suppl Spine):249-253, 2004

5. Branch CL, Branch CL Jr: Posterior lumbar interbody fusion with the keystone graft: technique and results. Surg Neurol 27:449-454, 1987

6. Ceylan D, Yaldiz C, Asil K, Kaçira T, Tatarli N, Can A: Intraoperative antepulsion of a posterior lumbar interbody fusion cage: three case reports. Pan Afr Med J 20:342, 2015

7. Chen CL, Chou CW, Su WW, Cheng CY, Yu CT: Dislodged upper thoracic cage in the gastrointestinal tract: a case report and literature reviews. Spine (Phila Pa 1976) 33:E802E806, 2008

8. Chen L, Yang H, Tang T: Cage migration in spondylolisthesis treated with posterior lumbar interbody fusion using BAK cages. Spine (Phila Pa 1976) 30:2171-2175, 2005

9. Enker P, Steffee AD: Interbody fusion and instrumentation. Clin Orthop Relat Res (300):90-101, 1994

10. Faro FD, Farnsworth CL, Shapiro GS, Mohamad F, White $\mathrm{KK}$, Breisch E, et al: Thoracic vertebral screw impingement on the aorta in an in vivo bovine model. Spine (Phila Pa 1976) 30:2406-2413, 2005

11. Foxx KC, Kwak RC, Latzman JM, Samadani U: A retrospective analysis of pedicle screws in contact with the great vessels. J Neurosurg Spine 13:403-406, 2010

12. Jendrisak MD: Spontaneous abdominal aortic rupture from erosion by a lumbar spine fixation device: a case report. Surgery 99:631-633, 1986

13. Kimura H, Shikata J, Odate S, Soeda T, Yamamura S: Risk factors for cage retropulsion after posterior lumbar interbody fusion: analysis of 1070 cases. Spine (Phila Pa 1976) 37:1164-1169, 2012

14. Kopp R, Beisse R, Weidenhagen R, Piltz S, Hauck S, Becker $\mathrm{CR}$, et al: Strategies for prevention and operative treatment of aortic lesions related to spinal interventions. Spine (Phila Pa 1976) 32:E753-E760, 2007

15. Kueper J, Fantini GA, Walker BR, Aichmair A, Hughes AP: Incidence of vascular complications during lateral lumbar interbody fusion: an examination of the mini-open access technique. Eur Spine J 24:800-809, 2015

16. Lin PM, Cautilli RA, Joyce MF: Posterior lumbar interbody fusion. Clin Orthop Relat Res (180):154-168, 1983

17. Lowe TG, Tahernia AD, O'Brien MF, Smith DA: Unilateral transforaminal posterior lumbar interbody fusion (TLIF): indications, technique, and 2-year results. J Spinal Disord Tech 15:31-38, 2002

18. McLaughlin MR, Haid RW Jr, Rodts GE Jr, Subach BR: Posterior lumbar interbody fusion: indications, techniques, and results. Clin Neurosurg 47:514-527, 2000

19. Ohnishi T, Neo M, Matsushita M, Komeda M, Koyama T, Nakamura T: Delayed aortic rupture caused by an implanted anterior spinal device. Case report. J Neurosurg 95 (2 Suppl):253-256, 2001

20. Papadoulas S, Konstantinou D, Kourea HP, Kritikos N, Haftouras N, Tsolakis JA: Vascular injury complicating lumbar disc surgery. A systematic review. Eur J Vasc Endovasc Surg 24:189-195, 2002 
21. Pawar UM, Kundnani V, Nene A: Major vessel injury with cage migration: surgical complication in a case of spondylodiscitis. Spine (Phila Pa 1976) 35:E663-E666, 2010

22. Proubasta IR, Vallvé EQ, Aguilar LF, Villanueva CL, Iglesias $\mathrm{JJ}$ : Intraoperative antepulsion of a fusion cage in posterior lumbar interbody fusion: a case report and review of the literature. Spine (Phila Pa 1976) 27:E399-E402, 2002

23. Suk SI, Lee CK, Kim WJ, Lee JH, Cho KJ, Kim HG: Adding posterior lumbar interbody fusion to pedicle screw fixation and posterolateral fusion after decompression in spondylolytic spondylolisthesis. Spine (Phila Pa 1976) 22:210-220, 1997

24. Yip SL, Woo SB, Kwok TK, Mak KH: Nightmare of lumbar diskectomy: aorta laceration. Spine (Phila Pa 1976) 36:E1758-E1760, 2011

25. Yoshimoto H, Sato S, Nakagawa I, Hyakumachi T, Yanagibashi Y, Nitta F, et al: Deep vein thrombosis due to migrated graft bone after posterior lumbosacral interbody fusion. Case report. J Neurosurg Spine 6:47-51, 2007

\section{Disclosures}

The authors report no conflict of interest concerning the materi- als or methods used in this study or the findings specified in this paper.

\section{Author Contributions}

Conception and design: Oshima, Murase, Takeshita, Miyoshi, Kunogi, Yamazaki, Ariyoshi, Sano, Hirohiko, Takeshita, Tanaka. Acquisition of data: all authors. Analysis and interpretation of data: Oshima, Murase. Critically revising the article: Oshima, Murase. Reviewed submitted version of manuscript: Oshima, Murase. Approved the final version of the manuscript on behalf of all authors: Oshima. Administrative/technical/material support: Oshima, Murase, Takeshita. Study supervision: Oshima, Murase, Takeshita, Tanaka.

\section{Correspondence}

Yasushi Oshima, Department of Orthopaedic Surgery, University of Tokyo, 7-3-1 Hongo, Bunkyo-ku, Tokyo 113-0033, Japan. email: yoo-tky@umin.ac.jp. 